\title{
LuxS-dependent quorum sensing in Porphyromonas gingivalis modulates protease and haemagglutinin activities but is not essential for virulence
}

\author{
Nicola A. Burgess, ${ }^{1,2}$ David F. Kirke, ${ }^{1}$ Paul Williams, ${ }^{1,2}$ Klaus Winzer, ${ }^{2}$ \\ Kim R. Hardie, ${ }^{1,2}$ Nicholas L. Meyers, ${ }^{3} \dagger$ Joseph Aduse-Opoku, ${ }^{4}$ \\ Michael A. Curtis ${ }^{4}$ and Miguel Cámara ${ }^{1,2}$
}

1 Institute of Infections and Immunity, Queens Medical Centre, University of Nottingham, Nottingham NG7 2UH, UK

2 School of Pharmaceutical Sciences, University of Nottingham, University Park, Nottingham NG7 2RD, UK

3 SmithKline Beecham Pharmaceuticals, Harlow CM19 5AW, UK

4 MRC Molecular Pathogenesis Group, Department of Oral Microbiology, St Bartholomews and the Royal London School of Dentistry, 32 Newark St, London E1 2AA, UK

\author{
Author for correspondence: Paul Williams. Tel: +44 115 9515047. Fax: +44 1158466296. \\ e-mail: paul.williams@nottingham.ac.uk
}

\begin{abstract}
Porphyromonas gingivalis is a Gram-negative black-pigmented obligate anaerobe implicated in the aetiology of human periodontal disease. The virulence of $\boldsymbol{P}$. gingivalis is associated with the elaboration of the cysteine proteases Arg-gingipain (Rgp) and Lys-gingipain (Kgp), which are produced at high bacterial cell densities. To determine whether quorum sensing plays a role in the regulation of Rgp and Kgp, biosensors capable of detecting either $\mathbf{N}$ acylhomoserine lactone (AHLs) or the luxS-dependent autoinducer (AI-2) quorum-sensing signalling molecules in spent culture supernatants were first employed. While no AHLs could be detected, the Vibrio harveyi BB170 biosensor was activated by spent $\boldsymbol{P}$. gingivalis W50 culture supernatants. The $P$. gingivalis luxS gene was cloned and demonstrated to restore AI-2 production in the Escherichia coli luxS mutant DH5 $\alpha$. Mutation of luxS abolished AI-2 production in $\boldsymbol{P}$. gingivalis. Western blotting using antibodies raised against the recombinant protein revealed that LuxS levels increased throughout growth even though Al-2 activity was only maximally detected at the midexponential phase of growth and disappeared by the onset of stationary phase. Similar results were obtained with $E$. coli DH5 $\alpha$ transformed with luxS, suggesting that Al-2 production is not limited by a lack of LuxS protein. Analysis of Rgp and Kgp protease activities revealed that the $P$. gingivalis luxS mutant produced around $45 \%$ less Rgp and $30 \%$ less Kgp activity than the parent strain. In addition, the luxS mutant exhibited a fourfold reduction in haemagglutinin titre. However, these reductions in virulence determinant levels were insufficient to attenuate the luxS mutant in a murine lesion model of $P$. gingivalis infection.
\end{abstract}

Keywords: Porphyromonas gingivalis, $\operatorname{lu} x S$, quorum sensing, gingipains, haemagglutinins

\section{INTRODUCTION}

Porphyromonas gingivalis is found in the subgingival plaques of patients with progressive periodontitis. This black-pigmented Gram-negative anaerobe has been strongly implicated in the aetiology of this chronic

†Present address: Alizyme, Granta Park, Great Abington, Cambridge CB1 6GS, UK.

Abbreviations: AHLs, $\mathrm{N}$-acylhomoserine lactones; Al-2, autoinducer 2. inflammatory disease which ultimately leads to the loss of tooth-supporting tissues and periodontal bone resorption. $P$. gingivalis has also been implicated as a risk factor for other conditions including cardiovascular disease and pre-term delivery of low birth weight infants (Beck et al., 1996; Offenbacher et al., 1996; Lamont \& Jenkinson, 1998).

In the periodontal pocket, $P$. gingivalis is found predominantly as a component of complex biofilms containing multiple bacterial species. As well as a capacity 
to adhere to a variety of host cell surfaces including the teeth, gingiva, cheek and tongue, $P$. gingivalis also adheres to other oral bacteria, presumably to facilitate colonization and survival within the oral cavity. $P$. gingivalis produces multiple cell envelope and exoproduct virulence determinants capable of promoting attachment, degrading host cell tissues and immune effector molecules, acquiring nutrients and facilitating epithelial cell invasion (Holt et al., 1999; Kinder \& Holt, 1989; Lamont \& Jenkinson, 1998; Yoneda \& Kuramitsu, 1996). Probably the most intensively investigated $P$. gingivalis virulence determinants are the exoproteases (Aduse-Opoku et al., 1995; Kuramitsu et al., 1997; Nakayama et al., 1995; Okamoto et al., 1996). Two major classes of cysteine proteinases specific for Arg-X and Lys-X bonds have been characterized and termed Arg-gingipain (Rgp) and Lys-gingipain (Kgp), respectively (Curtis et al., 1999). The multiple protease forms present on the $P$. gingivalis cell surface and in spent culture supernatants arise from the products of three genes: $r g p A$ and $r g p B$ (which encode the Rgp activity) and $k g p$ (which encodes the Kgp activity). The different forms appear to arise from the proteolytic posttranslational processing of larger polyprotein precursors (Curtis et al., 1999). For example, for RgpA, the initial precursor consisting of a pro-region, $\alpha$-catalytic domain, a $\beta$-adhesin/haemagglutinin domain and a C-terminal $\gamma$ domain is cleaved to generate three mature isoenzyme forms, HRgpA (an $\alpha \beta$-heterodimer of around $110 \mathrm{kDa}$ ), $\operatorname{RgpA}_{\text {cat }}(\alpha$ monomer of approx. $55 \mathrm{kDa})$ and mt$\mathrm{RgpA}_{\text {cat }}$ (a highly post-translationally modified $\alpha$ monomer of $70-80 \mathrm{kDa}$ ). These proteases degrade a variety of host proteins including immunoglobulins, complement cascade components, collagen, fibrinogen and fibronectin. As a consequence, they not only promote resistance to host defence mechanisms but also generate free amino acids and small peptides for use as nutrients (Shah \& Gharbia, 1989). Furthermore, the haemagglutinin/adhesin domains of RgpA and Kgp share significant amino acid sequence homology with haemagglutinin domain of HagA, a protein containing four contiguous direct repeats each of which contains a functional haemagglutinin domain (Han et al., 1996). P. gingivalis also possesses four further haemagglutinin genes (hagB, hagC, hagD and hagE; Lépine et al., 1996), and the ability to agglutinate erythrocytes strongly is a feature which distinguishes the organism from other black-pigmented anaerobes (Mayrand \& Holt, 1988). Although the functions and contribution of these multiple haemagglutinins to virulence are not well understood, they clearly promote adherence and colonization of tissue surfaces and, in conjunction with the exoproteases, promote attachment and detachment to target human cells (Chen et al., 2001).

To facilitate adaptation to life within the oral cavity, $P$. gingivalis must be capable of sensing and responding to the prevailing environmental conditions, including variations in temperature, oxygen tension, $\mathrm{pH}$, nutrient availability and the presence of other cells. While there is relatively little information on the mechanisms by which gene expression is regulated in this strict anaerobe, it is clear that the levels of virulence factors such as the proteases, fimbriae and haemagglutinins are controlled by temperature and haemin availability and affected by the presence of serum and saliva (Genco et al., 1995; Tokuda et al., 1998; Xie et al., 2000). Regulation has been shown to occur at both transcriptional and post-transcriptional levels and intriguingly the fimbrillin gene $(f i m A)$ has recently been suggested to be controlled by both the fimbrillin protein itself and by Rgp and Kgp (Xie et al., 2000). Given that Rgp and Kgp are produced maximally in the late stationary phase of growth (Rangarajan et al., 1997), it is possible that they are regulated in a cell-density-dependent manner via quorum sensing (Williams et al., 2000; Withers et al., 2001). This is a cell-to-cell communication mechanism which enables an individual bacterial cell to sense other bacterial cells and, in response, differentially express specific sets of genes. Quorum sensing involves the activation of a sensor or response regulator by a small diffusible signal molecule, and several chemically distinct families of such molecules have been identified. In Gram-negative bacteria, $N$-acylhomoserine lactones (AHLs) represent the most intensively investigated family of signal molecules, although a number of nonAHL molecules have also been described (Williams et al., 2000; Withers et al., 2001). These include a polar molecule termed AI-2, originally discovered in Vibrio harveyi as a second diffusible signal that could activate bioluminescence in the absence of $\mathrm{N}$-(3-hydroxybutanoyl)homoserine lactone (3-hydroxy-C4-HSL; Bassler et al., 1994). Although the structure of this diffusible signalling molecule has not yet been elucidated, a gene product (LuxS) involved in the production of AI- 2 has been identified not only in $V$. harveyi, but also in a number of human pathogens including Escherichia coli and Salmonella (Surette et al., 1999; Surette \& Bassler, 1998). However, the genes regulated directly via AI-2 in these Gram-negative pathogens have not yet been identified, although AI-2 has been reported to influence type III secretion in enterovirulent E. coli (Sperandio et al., 1999) and to modulate expression of virB in Shigella flexneri (Day \& Maurelli, 2001).

In the present paper, we have sought to determine whether $P$. gingivalis employs AHL- and/or AI-2/LuxSmediated quorum sensing. While we were unable to detect any AHLs, $P$. gingivalis was found to produce a signalling molecule(s) capable of activating a V. harveyibased AI-2 biosensor and to possess an orthologue of LuxS. Mutation of this gene resulted in the loss of AI-2 production and a reduction in protease and haemagglutinating activity which was insufficient to attenuate the luxS mutant in a murine lesion model of $P$. gingivalis infection.

\section{METHODS}

Bacteria, growth conditions and plasmids. The bacterial strains and plasmids used in this study are listed in Table 1.P. gingivalis W50 was maintained on Fastidious Anaerobic Agar (FAA; LabM) plates containing $5 \%$ horse blood (TCS) at 
Table 1. Bacterial strains and plasmids used in this study

\begin{tabular}{|c|c|c|}
\hline Strain/plasmid & Description & Source \\
\hline \multicolumn{3}{|l|}{ Strains } \\
\hline$P$. gingivalis $\mathrm{W} 50$ & Wild-type strain & ATCC 53978 \\
\hline P. gingivalis NBS1 & luxS mutant derived from W50 & This study \\
\hline$P$. gingivalis $\mathrm{K} 1 \mathrm{~A}$ & kgp mutant derived from W50 & $\begin{array}{l}\text { Aduse-Opoku et al. } \\
\text { (2000) }\end{array}$ \\
\hline V. harveyi BB120 & Wild-type strain & Bassler et al. (1994) \\
\hline V. harveyi $\mathrm{BB} 170$ & Mutant strain which responds to AI-2 & Bassler et al. (1994) \\
\hline C. violaceum $\mathrm{CV} 026$ & $\begin{array}{l}\text { Double Tn5 mutant derived from C. violaceum ATCC 31532; AHL sensor, } \\
\mathrm{Km}^{\mathrm{R}} \mathrm{Hg}^{\mathrm{R}}\end{array}$ & McClean et al. (1997) \\
\hline E. coli JM109(pSB401) & AHL biosensor containing a $\operatorname{lu} x R I^{\prime}:: C D A B E$ fusion in $\mathrm{pACYC184}, \mathrm{Tet}^{\mathrm{R}}$ & Winson et al. (1998) \\
\hline E. coli JM109(pSB536) & AHL biosensor containing an $a h y R I^{\prime}:: \operatorname{lu} x C D A B E$ fusion in $\mathrm{pRK} 415, \mathrm{Amp}^{\mathrm{R}}$ & Winson et al. (1998) \\
\hline E. coli JM109(pSB1075) & AHL biosensor containing a lasRI': luxCDABE fusion in $\mathrm{pUC18}, \mathrm{Tet}^{\mathrm{R}}$ & Winson et al. (1998) \\
\hline \multicolumn{3}{|l|}{ Plasmids } \\
\hline pBluescript SK $(+/-)$ & A phagemid derived from pUC19, $\mathrm{Amp}^{\mathrm{R}}$ & Stratagene \\
\hline pHG327 & A pUC8 derivative with the multiple cloning site of pUC18, $\mathrm{Amp}^{\mathrm{R}}$ & Stewart et al. (1986) \\
\hline pMal-c2 & High-level expression plasmid containing malE fused to $l a c Z \alpha, \mathrm{Amp}^{\mathrm{R}}$ & New England BioLabs \\
\hline pUC18Not & pUC18 containing Not $\mathrm{I}$ restriction sites flanking the multicloning region, Amp ${ }^{\mathrm{R}}$ & Herrero et al. (1990) \\
\hline pVA2198 & Plasmid containing ermF-ermAM for selection in both $P$. gingivalis and E. coli & Fletcher et al. (1995) \\
\hline pNB1 & A 989 bp fragment containing $\operatorname{luxS}$ and flanking regions ligated into pHG327 & This study \\
\hline pNB2 & Deletion of $231 \mathrm{bp}$ from $\operatorname{luxS}$ ligated into pHG327 & This study \\
\hline $\mathrm{pNB} 3$ & A 545 bp fragment containing luxS ligated into pMal-c2 & This study \\
\hline pHGin18 & Deletion of $231 \mathrm{bp}$ from luxS ligated into pUC18 & This study \\
\hline pGinerm & pHGin18 containing ermF-ermAM & This study \\
\hline
\end{tabular}

$37^{\circ} \mathrm{C}$ in an anaerobic cabinet equilibrated in $80 \%$ nitrogen $/ 10 \%$ hydrogen $/ 10 \%$ carbon dioxide. Liquid cultures of $P$. gingivalis W50 were grown anaerobically at $37^{\circ} \mathrm{C}$ in Brain Heart Infusion (BHI) broth (Oxoid) containing $5 \mu \mathrm{g}$ haemin $\mathrm{ml}^{-1}$. For detection of AI-2 in $P$. gingivalis culture supernatants, the organism was grown in the chemically defined medium described by Milner et al. (1996).

E. coli was grown at $37^{\circ} \mathrm{C}$ in Luria-Bertani (LB) medium and $V$. harveyi strains were grown at $30^{\circ} \mathrm{C}$ in Autoinducer Bioassay (AB) medium (Greenberg et al., 1979). Antibiotics, where required, were used at the following concentrations: ampicillin, $50 \mu \mathrm{g} \mathrm{ml}^{-1}$; clindamycin, $5 \mu \mathrm{g} \mathrm{ml}^{-1}$; tetracycline, $10 \mu \mathrm{g} \mathrm{m}^{-1}$; erythromycin, $5 \mu \mathrm{g} \mathrm{ml}^{-1}$; and kanamycin, $10 \mu \mathrm{g} \mathrm{ml}^{-1}$.

Bioassays for quorum-sensing signal molecules. Assays to detect the presence of AHLs were carried out as previously described (Cámara et al., 1998). Cell-free culture supernatants were extracted with dichloromethane and the extracts were subjected to TLC on reverse phase aluminium backed RP18 $\mathrm{F}_{254 \mathrm{~s}}$ TLC plates $(20 \mathrm{~cm} \times 20 \mathrm{~cm}$; Merck) with a mobile phase of $60 \%(\mathrm{v} / \mathrm{v})$ methanol in water. After chromatography, TLC plates were overlaid with soft top agar seeded with an AHL biosensor as described by McClean et al. (1997). The AHL biosensors used were the Tn5-generated Chromobacterium violaceum mutant CV026, which responds to a range of AHLs (with from $\mathrm{C}_{4}$ to $\mathrm{C}_{8}$ acyl side chains) by inducing the synthesis of the purple pigment violacein, and the lux-based biosensors (pSB401, pSB536 and pSB1075; Table 1) described by Winson et al. (1998), which are capable of detecting AHLs with acyl side chains from $\mathrm{C}_{4}$ to $\mathrm{C}_{14}$. Bioluminescence was detected using a Luminograph LB980 photon imaging camera (EG\&G Berthold).

The AI-2 bioassay, using the $V$. harveyi biosensor BB170
(luxN negative mutant), was carried out as described by Greenberg et al. (1979). Bacterial culture supernatants were added to the BB170 biosensor strain and AI-2 activity was measured in an EG\&G Wallac Victor ${ }^{2}$ luminometer as Relative Luminescence Units (RLU).

DNA manipulation techniques. Plasmid isolation, agarose electrophoresis, restriction digests and transformation of $E$. coli were performed as described by Sambrook et al. (1989). PCR amplifications were performed according to Ausubel et al. (1989). Bacterial chromosomal DNA was purified using the hexadecyltrimethylammonium bromide (CTAB) method (Murray \& Thompson, 1980). Purification of DNA fragments from agarose gels was carried out using Qiaquick DNA purification columns (Qiagen). Probes for Southern blot analysis were labelled using a DIG-labelling system (Boehringer Mannheim). Oligonucleotide synthesis and DNA sequencing was carried out at the Biopolymer Synthesis and Analysis Unit, Queen's Medical Centre, University of Nottingham.

Construction of a $P$. gingivalis genomic library in $E$. coli containing lux-based AHL reporters. To clone any putative $P$. gingivalis AHL synthases, the functional complementation assay described by Swift et al. (1993) was used. Genomic DNA from $P$. gingivalis W50 was digested with HindIII, KpnI, PstI, $B a m \mathrm{HI}$ or EcoRI, purified from agarose gels and ligated into pBluescript $\mathrm{SK}(+)$ previously digested with the appropriate restriction enzyme and dephosphorylated. The ligation mixtures were electroporated into E. coli JM109 containing either the lux-based AHL reporter pSB401 or pSB1075 (Winson et al., 1998; Laue et al., 2000) and plated on antibiotic selective media. Bioluminescent transformants were sought by viewing colonies with a Luminograph LB980 photon imaging camera (EG\&G Berthold). 
Cloning of luxS. For cloning of the P. gingivalis luxS gene, preliminary sequence data were obtained from The Institute for Genomic Research through the website at http://www. tigr.org. PCR amplification of $\operatorname{luxS}$ from $P$. gingivalis W50 chromosomal DNA was carried out using primers Por11 (5'GTATTATCAGCGGAATTCCCGGCGAAGGTCG-3') and Por12 (5'-GATACCGCCTCCGGATCCAATAATCCATCCGG-3'), which were designed using the published information on the $P$. gingivalis W83 genome sequence and contained EcoRI and BamHI restriction sites, respectively (see underlined sequences above). The PCR product was purified from an agarose gel and ligated into pHG327 (Table 1), previously digested with EcoRI and BamHI, to generate pNB1 (Table 1).

Generation of a $\boldsymbol{P}$. gingivalis luxS mutant. A deletion mutant of luxS lacking 231 internal nucleotides and containing an erythromycin (erm)-resistance cassette was constructed as follows. Two separate pairs of primers introducing NotI restriction sites (see underlined) were used: Por11 (see above) with Por13B (5'-GCGGCCGCCACCAAATGCTCGATCGTATGCCAG-3') (to amplify the $5^{\prime}$-end of $l u x S$ ) and Por14B (5'-TGGCGGCCGCGCGTGAGGTACTCGATGTAGCG-3') with Por12 (see above) (to amplify the $3^{\prime}$-end of luxS). P. gingivalis W50 chromosomal DNA was used as a template. Subsequently, each of the purified PCR products served as a template in a second PCR amplification using primers Por11 and Por12. The PCR product was digested with Bam HI and EcoRI and ligated into similarly digested pHG327 to generate pNB2 (Table 1). This plasmid was then digested with EcoRI and BamHI to release the truncated $\operatorname{lu} x S$ and ligated into pUC18, to generate pHGin18. To insert an erm cassette in the NotI site of the truncated $\operatorname{luxS}$, the erm cassette from pVA2198 (Rangarajan et al., 1997) was excised using EcoRI and BamHI digestion and cloned into pUC18Not, similarly digested. The erm cassette was then isolated from pUC18Not by digestion with NotI and subsequently cloned into the NotI site of pHGin18, thus creating pGinerm. This construct was electroporated into $P$. gingivalis W50 as described by Rangarajan et al. (1997), except that the plasmid was not linearized prior to electroporation. Transformants were selected on FAA containing clindamycin.

Overexpression, purification and detection of the $\boldsymbol{P}$. gingivalis LuxS. IuxS was amplified from $P$. gingivalis W50 chromosomal DNA, ligated into the EcoRI/BamHI sites of pMal-c2 (New England BioLabs) to generate pNB3, and electroporated into E. coli DH5 $\alpha$. The soluble recombinant MalE-LuxS fusion obtained was purified on an amylose resin column and bound protein was eluted with $10 \mathrm{mM}$ maltose. The purified MalE-LuxS fusion protein was cleaved using Factor Xa protease (New England BioLabs) and, following SDS-PAGE, the protein was electro-eluted and used to raise polyclonal antibodies in rabbits.

For the detection of recombinant or native LuxS protein, cell lysates from E. coli DH5 $\alpha, P$. gingivalis W50 and the luxS mutant were obtained by harvesting cells throughout the growth curve. The protease inhibitor $N^{\alpha}$-p-tosyl-L-lysine chloromethyl ketone (TLCK) was added, at a concentration of $50 \mathrm{mM}$, to $P$. gingivalis cells prior to lysis. Samples were standardized for protein content, boiled, resolved by SDSPAGE and transferred to nitrocellulose membranes. LuxS was detected by incubation with the LuxS polyclonal antiserum followed by a protein A-peroxidase conjugate and developed with an Amersham ECL kit (Amersham International).

Rgp and Kgp protease assays. Protease activity was measured as described by Rangarajan et al. (1997). P. gingivalis whole cultures, cell lysates (prepared by sonication of cells in PBS, $\mathrm{pH} 7 \cdot 4$ ) or cell-free supernatants prepared from cells grown to the same optical density $\left(\mathrm{OD}_{600}\right)$ were each added to a solution of $10 \mathrm{mM} \mathrm{L}$-cysteine, $10 \mathrm{mM} \mathrm{CaCl}_{2}, 100 \mathrm{mM}$ Tris/ $\mathrm{HCl}$ buffer (pH 8.1) containing $0.5 \mathrm{mM} \mathrm{N}$ - $\alpha$-benzoyl-DL-arginine- $p$-nitroanilide (DL-BApNA) or $0.5 \mathrm{mM} \mathrm{N}$ - $\alpha$-benzoylcarbonyl-L-lysine- $p$-nitroanilide (Ac-Lys- $p N A$ ). Samples were incubated for $1 \mathrm{~h}$ at $30^{\circ} \mathrm{C}$ and the reaction was monitored by following the increase in $A_{405}$ due to release of $p$-nitroaniline. Experiments were repeated three times in duplicate.

Haemagglutination assay. The haemagglutination assay was performed as described by Dusek et al. (1993). Essentially, sheep red blood cells (SRBCs) were harvested and washed twice in PBS ( $\mathrm{pH} \mathrm{7.4)}$ at $4{ }^{\circ} \mathrm{C}$ by centrifugation at $2000 \mathrm{~g}$ for $3 \mathrm{~min}$ and diluted to $0.5 \%(\mathrm{v} / \mathrm{v})$ in PBS. P. gingivalis W50 wild-type and luxS mutant were grown to an approximate $\mathrm{OD}_{580}$ of $0 \cdot 8$, washed once in PBS and resuspended to an $\mathrm{OD}_{580}$ of $1 \cdot 0$. Doubling dilutions of bacterial cells $(50 \mu \mathrm{l})$ were performed in V-shaped 96-well plates. The SRBCs $(50 \mu \mathrm{l})$ were added to the plates followed by incubation at $4{ }^{\circ} \mathrm{C}$ overnight and scored for haemagglutination.

$\boldsymbol{P}$. gingivalis murine infection model. The model described by Kesavalu et al. (1992) was used to determine whether the luxS mutant NBS1 exhibited reduced virulence. BALB/c mice (eight mice per group) were challenged s.c. for each strain with either $2.5 \times 10^{10}, 5 \times 10^{10}$ or $1 \times 10^{11}$ c.f.u. of $P$. gingivalis W50, the isogenic luxS mutant or the $k g p$ mutant K1A (Aduse-Opoku et al., 2000). K1A is known to be attenuated in this animal model (M. A. Curtis, unpublished data). For each strain, the mean survival time was determined for each of three challenge doses and the entire experiment was repeated twice. The data obtained were analysed statistically using standard methods for survival analysis allowing for right-censoring of time to death when animals survived to the scheduled end of the experiment. Life-table methods were used to display cumulative survival probabilities during experiments, while Cox proportional hazards models were used to estimate hazard ratios for comparisons of interest controlling for the effects of bacterial dose and for between-experiment variation in cumulative survival (Cox \& Oakes, 1984).

\section{RESULTS}

\section{AHL-dependent quorum sensing and $P$. gingivalis}

Solvent-extracted spent culture supernatants from $P$. gingivalis W50 were examined for the presence of AHLs by TLC assays employing four different biosensors capable of detecting a wide range of AHLs. No active spots were observed. Furthermore, interrogation of the $P$. gingivalis W83 genome sequence database (www. tigr.org) for homologues of (a) the LuxR transcriptional regulator family and (b) AHL synthases belonging to either the LuxI or LuxM family failed to identify any candidates. Since a putative third family of AHL synthases (HdtS) was recently described by Laue et al. (2000), the possible existence of a novel AHL synthase was investigated by introducing genomic libraries of $P$. gingivalis W50 DNA into E. coli strains containing either the lux-based AHL reporter pSB401 or pSB1075. Examination of each of the transformants obtained for bioluminescence and the characteristic accompanying satelliteism (where surrounding colonies emit light as a consequence of cross-feeding from a positive clone) proved negative. 
(a)

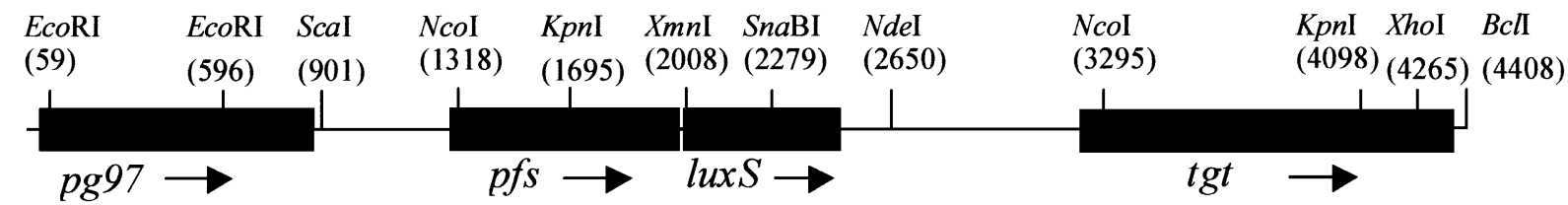

$1 \mathbf{k b}$

(b)

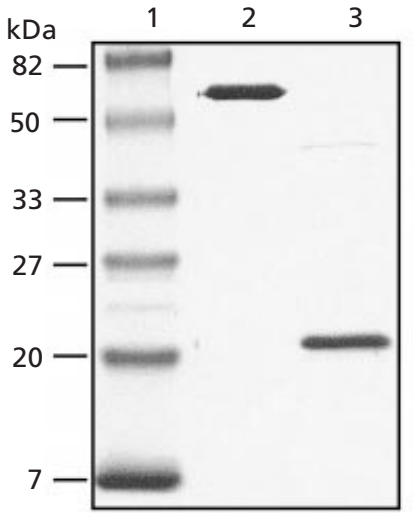

Fig. 1. (a) Diagrammatic representation of the $P$. gingivalis luxS locus showing upstream the presence of pfs and pg97, which encode a putative 5'-methylthioadenosine/S-adenosylhomocysteine nucleosidase and the $P$. gingivalis W50 $47 \mathrm{kDa}$ immunodominant antigen, respectively. Downstream of luxS is tgt, which is predicted to encode a tRNA guanine transglycosylase. (b) SDS-polyacrylamide gel showing the purification of the $P$. gingivalis LuxS protein. Lanes: 1 , molecular mass markers; 2, MalE-LuxS fusion purified by affinity chromatography eluted from an amylose resin column with maltose; 3 , purified LuxS protein cleaved from the MalE-LuxS fusion as described in Methods. The weak upper band in lane 3 is a dimer of LuxS.

\section{Characterization of a $P$. gingivalis LuxS orthologue}

To determine whether $P$. gingivalis possesses an AI$2 /$ LuxS-based quorum-sensing system, the $P$. gingivalis genome database was searched using the predicted amino acid sequence of the $V$. harveyi LuxS protein. A 159 amino acid residue $(18.5 \mathrm{kDa}) \mathrm{LuxS}$ orthologue was identified. When compared with other LuxS orthologues present in the databases, the $P$. gingivalis LuxS protein is most closely related to the LuxS of Borrelia burgdorferi (similarity $68 \%$; identity $50 \%$ ) and shares $33 \%$ identity (53\% similarity) with the E. coli LuxS protein. Examination of the DNA sequence surrounding $P$. gingivalis luxS (Fig. 1a) revealed the presence of an adjacent ORF, the translated amino acid sequence of which shares significant homology $(37 \%$ identity; $54 \%$ similarity) with the E. coli $p f s$ gene product, a $5^{\prime}$-methylthioadenosine/ $S$-adenosylhomocysteine nucleosidase (Cornell \& Riscoe, 1998). This $p f s$ gene is separated from luxS by only $24 \mathrm{bp}$, suggesting that the two genes constitute an operon. Homologues of the $P$. gingivalis W50 $47 \mathrm{kDa}$ immunodominant antigen (GenBank accession no. AF153770) and a tRNA guanine transglycosylase (Tgt; Reuter et al., 1991) were located approximately 700 bp upstream and downstream of the $p f s / l u x S$ locus, respectively (Fig. 1a).

To determine whether $\operatorname{luxS}$ encodes a functional protein, it was expressed in E. coli DH5 $\alpha$, which is AI-2 negative and carries a frameshift mutation in the $\operatorname{luxS}$ gene (Surette et al., 1999). This was achieved by amplification of luxS from P. gingivalis W50 by PCR, followed by cloning into pHG327 to generate pNB1. In contrast to $E$. coli $\mathrm{DH} 5 \alpha$, spent culture supernatants from E. coli DH5 $\alpha(\mathrm{pNB} 1)$ stimulated light production in the AI-2 biosensor strain $V$. harveyi BB170 (data not shown), suggesting that the recombinant $P$. gingivalis protein is functional. To confirm that the AI-2 activity detected was due to luxS expression, an internal fragment of $231 \mathrm{bp}$ was deleted and the truncated $\operatorname{luxS}$ gene was cloned into pHG237 to generate pNB2. Spent culture supernatants from E. coli DH5 $\alpha(\mathrm{pNB} 2)$ failed to stimulate bioluminescence in $V$. harveyi BB170 above background (data not shown).

\section{Al-2 levels in both $P$. gingivalis and $E$. coli do not correlate with LuxS levels}

To investigate the relationship between AI-2 levels and LuxS production as a function of growth in both E. coli and $P$. gingivalis, antibodies were first raised against the recombinant $P$. gingivalis LuxS protein expressed from pNB3 (Table 1) as a MalE-LuxS fusion. The $62 \mathrm{kDa}$ fusion protein was purified by amylose affinity chromatography, cleaved using Factor Xa and the recombinant LuxS protein obtained for antibody production via electro-elution of the protein from SDS-polyacrylamide gels used to separate LuxS from MalE (Fig. 1b).

E. coli $\mathrm{DH} 5 \alpha$ transformed with pNB3 (Table 1) produced AI-2, indicating either that the MalE-LuxS fusion protein was functional or that sufficient LuxS for AI-2 synthesis was cleaved by endogenous E. coli proteases. 
(a)

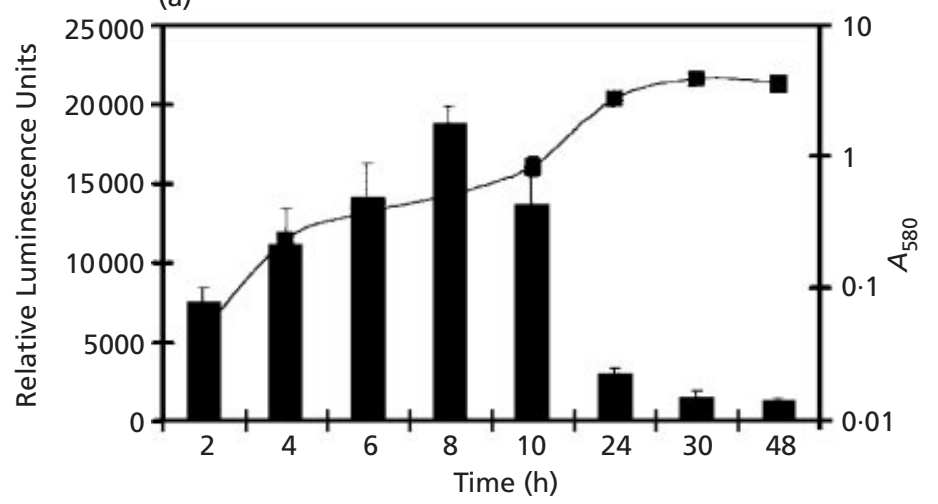

(b)

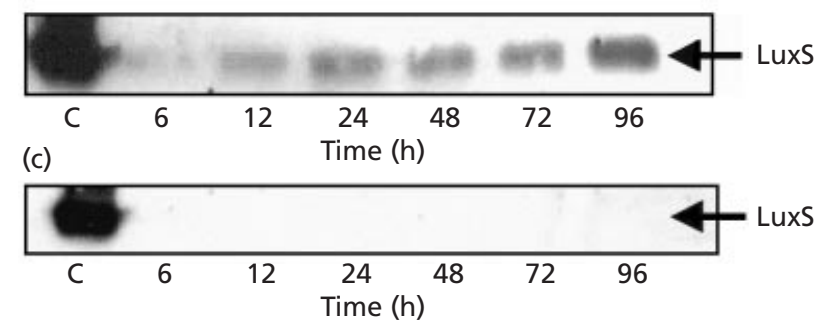

Fig. 2. (a) Growth and Al-2 production by $P$. gingivalis W50. $P$. gingivalis was grown anaerobically in defined medium for $48 \mathrm{~h}$ at $37^{\circ} \mathrm{C}$. At $2,4,6,8,10,24,30$ and $48 \mathrm{~h}$, cellfree culture supernatants were assayed for Al-2 activity using $V$. harveyi BB170. (b, c) Western blot analysis of whole-cell lysates of $P$. gingivalis W50 and the isogenic luxS mutant throughout the growth curve. Lane $C$ in each panel contains the purified recombinant LuxS fusion protein as a control and the remaining lanes contain lysates of W50 (b) or the luxS mutant (c) harvested throughout growth at 6, 12, 24, 48, 72 and $96 \mathrm{~h}$ of growth and adjusted to standardize protein content. Following Western blotting, membranes were probed with antibodies raised against the recombinant $P$. gingivalis LuxS.
Western blotting of E. coli DH5 $\alpha(\mathrm{pNB} 3)$ whole-cell extracts using antibodies raised against LuxS revealed that the LuxS protein accumulates throughout growth (data not shown). In addition, analysis of culture supernatants from E. coli $\mathrm{DH} 5 \alpha(\mathrm{pNB} 3)$ taken throughout the growth curve showed increasing levels of AI-2 activity from mid-exponential phase disappearing by early-stationary phase when LuxS levels are still increasing (data not shown). The levels of AI-2 production were higher when cells were grown in the presence of glucose $(0.4 \%, \mathrm{w} / \mathrm{v})$ than when this sugar was omitted (data not shown).

Bioluminescence assays using V. harveyi BB170 failed to reveal the presence of AI-2 in culture supernatants taken throughout the growth curve of $P$. gingivalis W50 grown in BHI medium. However, when this organism was grown in a chemically defined medium (DM), AI-2 was maximally detected at mid-exponential phase of growth (Fig. 2a), disappearing at the beginning of the stationary phase, while Western blots still detected increasing levels of LuxS (Fig. 2b). These results are consistent with those obtained in E. coli DH5 $\alpha$ (pNB3), suggesting that AI-2 production is not limited by LuxS protein levels.

\section{LuxS modulates protease levels and haemagglutination}

To determine whether LuxS contributes to the regulation of virulence determinants in $P$. gingivalis, a luxS null mutant (NBS1) was first constructed as described in Methods. Southern blot (data not shown) and Western blot analysis (Fig. 2c) confirmed the absence of LuxS protein and no AI-2 activity could be detected in spent culture supernatants of the luxS mutant using the $V$. harveyi BB170 AI-2 biosensor (data not shown).

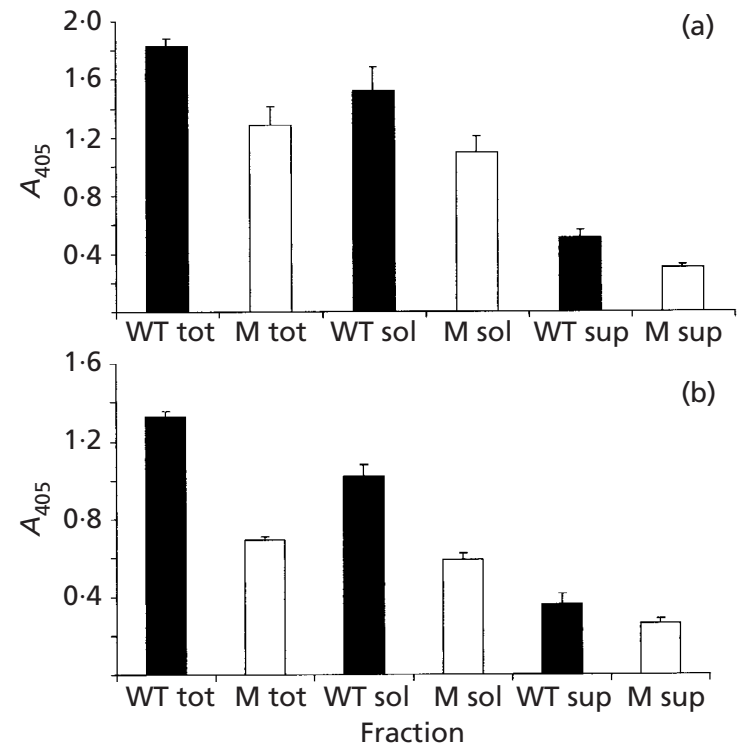

Fig. 3. Protease production by $P$. gingivalis $W 50$ and the isogenic luxS mutant. (a) Kgp and (b) Rgp levels were determined using the substrates Ac-Lys-pNA and BApNA, respectively, in whole cultures (tot), cell-free supernatants (sup) and lysates (sol) of $P$. gingivalis W50 wild-type (WT) and the luxS null mutant NBS1 (M). Fractions were prepared as described in Methods and enzyme activity was followed by incubation at $30^{\circ} \mathrm{C}$ for $1 \mathrm{~h}$. The reaction was monitored at 405 $\mathrm{nm}$.

To quantify the levels of protease activity produced by $P$. gingivalis W50 and the isogenic luxS mutant, whole culture, cell lysates and cell-free culture supernatants were incubated with either the Kgp substrate Ac-Lys$p \mathrm{NA}$ or the Rgp substrate DL-BApNA. Fig. 3(a) shows 
Table 2. Virulence of $P$. gingivalis in a murine soft tissue model

Values are the mean survival time $(\mathrm{h})$. Mice were challenged s.c. with either the $P$. gingivalis wild-type, luxS mutant (NBS1) or the kgp mutant K1A and examined up to $190 \mathrm{~h}$ for lesion formation, weight and lethality. The numbers of animals surviving are in parentheses.

\begin{tabular}{|c|c|c|c|}
\hline \multirow[t]{2}{*}{ Strain } & \multicolumn{3}{|c|}{ Challenge dose (c.f.u.) } \\
\hline & $2 \cdot 5 \times 10^{10}$ & $5 \times 10^{10}$ & $1 \times 10^{11}$ \\
\hline W50 & $190(8 / 8)$ & $31(0 / 8)$ & $22(0 / 8)$ \\
\hline NBS1 & $116(3 / 8)^{*}$ & $39(0 / 8)$ & $25(0 / 8)$ \\
\hline $\mathrm{K} 1 \mathrm{~A}$ & $190(8 / 8)$ & $113(2 / 8)$ & $64(0 / 8)$ \\
\hline
\end{tabular}

* Reduced survival time for the luxS mutant at this challenge dose is not statistically significant.

that after $48 \mathrm{~h}$ growth, each fraction prepared from the luxS mutant contained around $30 \%$ less Kgp activity than the parent strain. For Rgp, each fraction prepared from the $\operatorname{luxS}$ mutant contained around $45 \%$ less Rgp protease activity (Fig. 3b).

Since the C-terminal region of the RgpA family of proteinases shares extensive sequence homology with the haemagglutinins of $P$. gingivalis (Lamont $\&$ Jenkinson, 1998), we compared the capacity of the wildtype and $\operatorname{lu} x S$ mutant to agglutinate sheep erythrocytes. The haemagglutination titre of the luxS mutant (1 in 8) was fourfold lower than the wild-type ( 1 in 32 ; data not shown).

\section{luxS and the virulence of $P$. gingivalis}

$\mathrm{BALB} / \mathrm{c}$ mice were challenged s.c. with the wild-type, luxS mutant NBS1 or kgp mutant K1A. Table 2 shows that there were no differences in the mean survival times of mice infected with either the wild-type strain or $\operatorname{luxS}$ mutant. These data contrast with the kgp mutant, which was clearly attenuated as revealed by the extended survival times of mice inoculated with $5 \times 10^{10}$ or $1 \times 10^{11}$ c.f.u.

\section{DISCUSSION}

As a determinant of cell population density, quorum sensing is emerging as an integral component of bacterial global gene regulatory networks responsible for facilitating environmental adaptation. Several chemically distinct classes of quorum-sensing signal molecules have emerged, of which the AHL family in Gram-negative bacteria has been the most intensively investigated (Williams et al., 2000; Withers et al., 2001). Although we were unable to obtain any evidence to suggest that the Gram-negative anaerobe $P$. gingivalis employs AHLmediated quorum sensing, we have demonstrated that this periodontal pathogen produces a diffusible signal molecule capable of activating the $V$. harveyi AI-2 biosensor. During the preparation of this manuscript, two other groups also reported that $P$. gingivalis as well as other Gram-negative oral bacteria could activate the AI-2 biosensor (Frias et al., 2001; Chung et al., 2001). Production of AI-2 in $P$. gingivalis as determined using the $V$. harveyi biosensor BB170 is abolished following mutation of $\operatorname{luxS}$ (this study and Chung et al., 2001). Mutation of luxS in E. coli (Surette et al., 1999; Sperandio et al., 1999), Helicobacter pylori (Forsyth \& Cover, 2000; Joyce et al., 2000) and Shig. flexneri (Day $\&$ Maurelli, 2001) also results in the loss of AI-2 synthesis.

AI-2 appears to represent a new class of quorum-sensing signal molecule involved in cross-communication between different bacteria because Gram-negative and Gram-positive organisms both contain LuxS orthologues (Bassler et al., 1997; Bassler, 1999). However, at present, the chemical identity of the $V$. harveyi AI-2 molecule is not known although Schauder et al. (2001) have very recently reported that it is probably a furanone derived from 4,5-dihydroxy-2,3-pentadione and that the function of LuxS is to generate 4,5-dihydroxy-2,3pentadione from $S$-adenosylhomocysteine. Thus, in contrast to the AHLs, where a family of signal molecules with different $\mathrm{N}$-linked acyl chains has been identified (Withers et al., 2001), it has been suggested that AI-2 is structurally invariant, i.e. that despite their sequence divergence, LuxS orthologues all drive the synthesis of the same molecule (Schauder et al., 2001).

In $P$. gingivalis, the $l u x S$ gene is located adjacent to $p f_{s}$; RT-PCR analysis suggests that they constitute an operon (N. Burgess, unpublished data; Chung et al., 2001). The $p f s$ gene product is a $5^{\prime}$-methylthioadenosine/S-adenosylhomocysteine nucleosidase which can cleave the glycosidic bond releasing adenine and the corresponding thiopentose (either methylthioribose or $S$-ribosylhomocysteine; Cornell \& Riscoe, 1998). Indeed, during the preparation of this manuscript, Schauder et al. (2001) reported that AI-2 can be generated in vitro from the purified LuxS proteins of E. coli, V. harveyi, Vibrio cholerae, Salmonella typhimurium and Enterococcus faecalis in conjunction with Pfs and S-adenosylhomocysteine. We have also shown that the production of $\mathrm{AI}-2$ in vitro via the $P$. gingivalis LuxS protein and $S$-adenosylhomocysteine depends on the presence of the $P$. gingivalis $p f s$ gene product (Winzer et al., 2002).

By growing $P$. gingivalis in a chemically defined medium, we were able to show that AI-2 is produced during midexponential growth but appears to have been degraded by the stationary phase. These data are consistent with results obtained for E. coli DH5 $\alpha$ harbouring the $P$. gingivalis luxS gene (this paper) and with those of Surette et al. (1999) for Sal. typhimurium. Similarly, in H. pylori, AI-2 activity is maximal in early-exponential phase and rapidly decreases as the organism enters stationary phase (Joyce et al., 2000). These data suggest that LuxS-mediated quorum sensing is only operational during periods of rapid, nutrient-rich growth. Interestingly, Western blot analysis of LuxS production in $P$. 
gingivalis and E. coli throughout the growth curve revealed that despite the LuxS protein being present, AI2 activity is absent from stationary phase samples. In both E. coli and Sal. typhimurium, the existence of a degradation pathway has been proposed, given the rapid loss of AI-2 when carbohydrate is depleted from the medium or when the cells enter stationary phase (Surette \& Bassler, 1998). This implies that the substrates necessary for AI-2 synthesis are not available in stationary phase and/or that the levels of LuxS are maintained to enable the cells to take immediate advantage of any changes in environmental parameters (e.g. nutrient availablity) without the need for transcription (Surette \& Bassler, 1999). Alternatively, the retention of the LuxS protein may be required to prevent accumulation of $S$-ribosylhomocysteine, which is an inhibitor of all $S$-adenosylmethionine-dependent methylation reactions (Cornell \& Riscoe, 1998), or to function as a component of a salvaging pathway for adenine and methionine synthesis.

Apart from modulation of bioluminescence in $V$. harveyi (Bassler et al., 1994), the identification and characterization of the signal transduction pathways and target genes of the AI-2 quorum-sensing system in other bacteria has met with limited success. For H. pylori, mutation of $\operatorname{lu} x S$ did not influence expression of any of the established virulence factors (e.g. vacA) and analysis of the two-dimensional protein profiles of wild-type and mutant failed to reveal any differences (Joyce et al., 2000; Forsyth \& Cover, 2000). In enteropathogenic E. coli, Sperandio et al. (1999) reported that LuxS weakly regulates operons encoding the type III secretion apparatus, intimin receptor and intimin. Although the virulence of pathogenic E. coli luxS mutants was not evaluated, an Shig. flexneri luxS mutant was discovered to be as invasive and virulent as the parental strain despite the contribution of LuxS in enhancing expression of $\operatorname{vir} B$ in the late-exponential phase (Day \& Maurelli, 2001). In P. gingivalis, Chung et al. (2001) have very recently used the qualitative techniques of differential display (DD)-PCR and RT-PCR to show that mutation of luxS influenced the transcript levels of several genes involved in haemin acquisition and an exinuclease $\mathrm{ABC}$ homologue. Although their analysis of $\operatorname{rgp} A$ expression gave equivocal results depending on which technique was used, Chung et al. (2001) reported that rgpA transcript levels were increased in the luxS mutant. However, no data were shown and no direct quantitative assays of Rgp protease activity were undertaken. In the present paper, we have established that mutation of $\operatorname{luxS}$ in $P$. gingivalis results in a $30-45 \%$ reduction in the levels of the Rgp and Kgp proteases. While this may at first appear to conflict with the results of Chung et al. (2001), a $30 \%$ reduction in protein is unlikely to be accompanied by a reduction in transcript level of sufficient magnitude to be detectable by DD-PCR or by RT-PCR. It is also possible that the regulation is at the post-transcriptional level. Both RgpA and Kgp are subject to significant post-translational processing (Curtis et al., 1999) which may also be influenced by the
luxS mutation. Indeed, preliminary analysis of the Rgp isoforms in the $\operatorname{luxS}$ mutant NBS1 revealed a reduction in the level of the membrane-type RgpB isoform ( $\mathrm{mt}$ RgpB; unpublished data), the main vesicle-associated form of the Arg-gingipains (Aduse-Opoku et al., 1998). This suggests that LuxS may influence the post-translational modification pathway of these enzymes.

Furthermore, the $\operatorname{luxS}$ mutant exhibits reduced wholecell haemagglutinin activity compared with the parent. This finding is consistent with previous reports that cysteine proteases and haemagglutinin activities of $P$. gingivalis appear to be structurally related (Pavloff et al., 1995 ; Yoneda \& Kuramitsu, 1996). This phenotype may therefore be associated with RgpA and Kgp, which contain haemagglutinin/adhesion domains (Curtis et al., 1999). Alternatively, it may be a consequence of reduced expression of haemagglutinins such as hagA, which encodes a surface protein haemagglutinin containing domains homologous to the adhesion domains of Rgp and Kgp. Thus given the central role of proteases in the pathogenicity of $P$. gingivalis, we sought to determine whether the reduced production of Rgp and Kgp by the $\operatorname{lu} x S$ mutant influenced virulence. In an acute mouse model of soft tissue infection, there were no obvious differences in the virulence of the $P$. gingivalis luxS mutant when compared with the parent strain. However, although frequently used to assess the virulence of, and immunity to, $P$. gingivalis, the infection model used (Kesavalu et al., 1992) depends on a high bacterial challenge dose and does not follow the natural course of periodontal disease. In addition, $P$. gingivalis is generally found only in low numbers within the oral cavity such that under these conditions the expression of proteases/haemagglutinins may be reduced. In disease, $P$. gingivalis can represent a significant proportion of the total flora and, under these conditions, it remains possible that AI-2/LuxS may a play a role in upregulation of these virulence properties and hence the development of disease.

\section{ACKNOWLEDGEMENTS}

We would like to thank Helen Evans (Department of Oral Microbiology, St Bartholomews and the Royal London School of Dentistry) for technical assistance with the murine infection model and Dr Jonathan Stern (Department of Social Medicine, University of Bristol, UK) for statistical analysis of the infection model data. The work presented was supported by a joint Biotechnology and Biological Sciences Research Council, UK and SmithKline Beecham Pharmaceuticals CASE studentship and by grants from the Medical Research Council UK.

\section{REFERENCES}

Aduse-Opoku, J., Muir, J., Slaney, J. M., Rangarajan, M. \& Curtis, M. A. (1995). Characterization, genetic analysis and expression of a protease antigen (PrpRI) of Porphyromonas gingivalis W50. Infect Immun 63, 4744-4754.

Aduse-Opoku, J., Rangarajan, M., Young, K. A. \& Curtis, M. A. (1998). Maturation of the arginine-specific proteases of Por- 
phyromonas gingivalis W50 is dependent on a functional prR2 protease gene. Infect Immun 66, 1594-1600.

Aduse-Opoku, J., Davies, N. N., Gallagher, A., Hashim, A., Evans, H. E. A., Rangarajan, M., Slaney, J. M. \& Curtis, M. A. (2000). Generation of Lys-gingipain protease activity in Porphyromonas gingivalis W50 is independent of Arg-gingipain protease activities. Microbiology 146, 1933-1940.

Ausubel, F. M., Brent, R., Kingston, R. E., Moore, D. D., Seidman, J. G., Smith, J. A. \& Struhl, K. (1989). Short Protocols in Molecular Biology. Chichester: Wiley.

Bassler, B. L. (1999). How bacteria talk to each other: regulation of gene expression by quorum sensing. Curr Opin Microbiol 2, 582-587.

Bassler, B. L., Wright, M. \& Silverman, M. R. (1994). Multiple signalling systems controlling expression of luminescence in Vibrio harveyi: sequence and function of genes encoding a second sensory pathway. Mol Microbiol 13, 273-286.

Bassler, B. L., Greenberg, E. P. \& Stevens, A. M. (1997). Crossspecies induction of luminescence in the quorum sensing bacterium Vibrio harveyi. J Bacteriol 179, 4043-4045.

Beck, J., Garcia, R., Heiss, G., Vokonas, P. S. \& Offenbacher, S. (1996). Periodontal disease and cardiovascular disease. J Periodontol 67, 1123-1137.

Cámara, M., Daykin, M. \& Chhabra, S. R. (1998). Detection, purification and synthesis of $\mathrm{N}$-acyl homoserine lactone quorum sensing molecules. Methods Microbiol 27, 319-330.

Chen, T., Nakayama, K., Belliveau, L. \& Duncan, M. J. (2001). Porphyromonas gingivalis gingipains and adhesion to epithelial cells. Infect Immun 69, 3048-3056.

Chung, W. O., Park, Y., Lamont, R. J., McNab, R., Barbieri, B. \& Demuth, D. R. (2001). Signaling system in Porphyromonas gingivalis based on a LuxS protein. J Bacteriol 183, 3903-3909.

Cornell, K. A. \& Riscoe, M. K. (1998). Cloning and expression of Escherichia coli 5'-methylthioadenosine/S-adenosylhomocysteine nucleosidase: identification of the $p f s$ gene product. Biochim Biophys Acta 1396, 8-14.

Cox, D. R. \& Oakes, D. (1984). Analysis of Survival Data. London: Chapman and Hall.

Curtis, M. A., Kuramitsu, H. K., Lantz, M., Macrina, F. L., Nakayama, K., Potempa, J., Reynolds, E. C. \& Aduse-Opoku, J. (1999). Molecular genetics and nomenclature of proteases of Porphyromonas gingivalis. J Periodontal Res 34, 464-472.

Day, W. A. \& Maurelli, A. T. (2001). Shigella flexneri LuxS quorum sensing system modulates $\operatorname{vir} B$ expression but is not essential for virulence. Infect Immun 69, 15-23.

Dusek, D. M., Progulske-Fox, A., Whitlock, J. \& Brown, T. A. (1993). Isolation and characterization of a cloned Porphyromonas gingivalis hemagglutinin from an avirulent strain of Salmonella typhimurium. Infect Immun 61, 940-946.

Fletcher, H. M., Schenkein, H. A. \& Macrina, F. L. (1995). Cloning and characterisation of a new protease gene (prtH) from Porphyromonas gingivalis. Infect Immun 62, 4279-4286.

Forsyth, M. H. \& Cover, T. L. (2000). Intercellular communication in Helicobacter pylori: $\operatorname{lu} x S$ is essential for the production of an extracellular signaling molecule. Infect Immun 68, 3193-3199.

Frias, J., Olle, E. \& Alsina, M. (2001). Periodontal pathogens produce quorum sensing signal molecules. Infect Immun 69, 3431-3434.

Genco, C. A., Simpson, W., Forng, R. Y., Egal, M. \& Odusanya, B. M. (1995). Characterization of a Tn4351-generated hemin uptake mutant of Porphyromonas gingivalis: evidence for the co- ordinate regulation of virulence factors by hemin. Infect Immun 63, 2459-2466.

Greenberg, P., Hastings, J. W. \& Ulitzur, S. (1979). Induction of luciferase synthesis in Beneckea harveyi by other marine bacteria. Arch Microbiol 120, 87-91.

Han, N., Whitlock, J. \& Progulske-Fox, A. (1996). The hemagglutinin gene A (hagA) of Porphyromonas gingivalis 381 contains four large contiguous direct repeats. Infect Immun 64, 4000-4007.

Herrero, M., de Lorenzo, V. \& Timmis, K. N. (1990). Transposon vectors containing non-antibiotic resistance selection markers for cloning and stable chromosomal insertion of foreign genes in gram-negative bacteria. J Bacteriol 172, 6557-6567.

Holt, S. C., Kesavalu, L., Walker, S. \& Genco, C. A. (1999). Virulence factors of Porphyromonas gingivalis. Periodontol 2000 20, 168-238.

Joyce, E. A., Bassler, B. L. \& Wright, A. (2000). Evidence for a signaling system in Helicobacter pylori: detection of a luxSencoded autoinducer. J Bacteriol 182, 3638-3643.

Kesavalu, L., Ebersole, J. L., Machen, R. L. \& Holt, S. C. (1992). Porphyromonas gingivalis virulence in mice: induction of immunity to bacterial components. Infect Immun 60, 1455-1464.

Kinder, S. A. \& Holt, S. C. (1989). Characterization of coaggregation between principal periodontal pathogens. Infect Immun 34, $757-765$.

Kuramitsu, H. K., Tokuda, M., Yoneda, M., Duncan, M. \& Cho, M. I. (1997). Multiple colonization defects in cysteine protease mutant of Porphyromonas gingivalis. J Periodontal Res 32, 140-142.

Lamont, R. J. \& Jenkinson, H. F. (1998). Life below the gum line: pathogenic mechanisms of Porphyromonas gingivalis. Microbiol Mol Biol Rev 62, 1244-1263.

Laue, B. E., Jiang, Y., Chhabra, S. R., Jacob, S., Stewart, G. S. A. B., Hardman, A., Downie, J. A., O'Gara, F. \& Williams, P. (2000). The biocontrol strain Pseudomonas fluorescens F113 produces the Rhizobium small bacteriocin, $N$-(3-hydroxy-7-cis-tetradecenoyl)homoserine lactone, via $\mathrm{HdtS}$, a putative novel $\mathrm{N}$-acylhomoserine lactone synthase. Microbiology 146, 2469-2480.

Lépine, G., Ellen, R. P. \& Progulske-Fox, A. (1996). Construction and preliminary characterization of three hemagglutinin mutants of Porphyromonas gingivalis. Infect Immun 64, 1467-1472.

McClean, K. H., Winson, M. K., Fish, L. \& 9 other authors (1997). Quorum sensing and Chromobacterium violaceum: exploitation of violacein production and inhibition for the detection of $\mathrm{N}$ acylhomoserine lactones. Microbiology 143, 3703-3711.

Mayrand, D. \& Holt, S. C. (1988). Biology of asaccharolytic blackpigmented Bacteroides species. Microbiol Rev 52, 134-152.

Milner, P., Batten, J. E. \& Curtis, M. A. (1996). Development of a simple chemically defined medium for Porphyromonas gingivalis: requirement for alpha-ketoglutarate. FEMS Microbiol Lett 140, 125-130.

Murray, M. G. \& Thompson, W. F. (1980). Rapid isolation of highmolecular-weight plant DNA. Nucleic Acids Res 8, 4321-4325.

Nakayama, K., Kadowaki, T., Okamoto, K. \& Yamamoto, T. (1995). Construction and characterization of arginine-specific cysteine proteinase (Arg-gingipain)-deficient mutants of Porphyromonas gingivalis. J Biol Chem 270, 23619-23626.

Offenbacher, S., Katz, V., Fertik, G., Collins, J., Boyd, D., Maynor, G., McKaig, R. \& Beck, J. (1996). Periodontal infection as a possible risk factor for preterm low birth weight. J Periodontol 67, 1103-1113.

Okamoto, K., Kadowaki, T., Nakayama, K. \& Yamamoto, K. 
(1996). Cloning and sequencing of the gene encoding a novel lysine-specific cysteine proteinase (Lys-gingipain) in Porphyromonas gingivalis: structural relationship with the argininespecific cysteine proteinase (Arg-gingipain). J Biochem 120, 398-406.

Pavloff, N., Potempa, J., Pike, R. N., Prochazka, V., Kiefer, M. C., Travis, J. \& Barr, P. J. (1995). Molecular cloning and structural characterization of the arg-gingipain proteinase of Porphyromonas gingivalis - biosynthesis as a proteinase adhesion polyprotein. J Biol Chem 270, 1007-1010.

Rangarajan, M., Aduse-Opoku, J., Slaney, J. M., Young, K. A. \& Curtis, M. A. (1997). The $p r p R 1$ and $p r R 2$ arginine-specific protease genes of Porphyromonas gingivalis W50 produce five biochemically distinct enzymes. Mol Microbiol 23, 955-965.

Reuter, K., Slany, R., Ullrich, F. \& Kersten, H. (1991). Structure and organization of Escherichia coli genes involved in biosynthesis of the deazaguanine derivative queuine, a nutrient factor for eukaryotes. J Bacteriol 173, 2256-2264.

Sambrook, J., Fritsch, E. F. \& Maniatis, T. (1989). Molecular Cloning: a Laboratory Manual, 2nd edn. Cold Spring Harbor, NY : Cold Spring Harbor Laboratory.

Schauder, S., Shokat, K., Surette, M. G. \& Bassler, B. L. (2001). The LuxS family of bacterial autoinducers: biosynthesis of a novel quorum sensing signal molecule. Mol Microbiol 41, 463-476.

Shah, H. N. \& Gharbia, S. E. (1989). Ecological events in subgingival dental plaque with reference to Bacteroides and Fusobacterium species. Infection 17, 264-268.

Sperandio, V., Mellies, J. L., Nguyen, W., Shin, S. \& Kaper, J. B. (1999). Quorum sensing controls expression of the type III secretion gene transcription and protein secretion in enterohemorrhagic and enteropathogenic Escherichia coli. Proc Natl Acad Sci U S A 96, 15196-15201.

Stewart, G. S. A. B., Lubinsky-Mink, S., Jackson, C. G., Cassel, A. \& Kuhn, J. (1986). pHG165 : a pBR322 copy number derivative of pUC8 for cloning and expression. Plasmid 15, 172-181.

Surette, M. G. \& Bassler, B. L. (1998). Quorum sensing in Escherichia coli and Salmonella typhimurium. Proc Natl Acad Sci U S A 95, 7046-7050.
Surette, M. G. \& Bassler, B. L. (1999). Regulation of autoinducer production in Salmonella typhimurium. Mol Microbiol 31, 585-595.

Surette, M. G., Miller, M. B. \& Bassler, B. L. (1999). Quorum sensing in Escherichia coli, Salmonella typhimurium, and Vibrio harveyi: a new family of genes responsible for autoinducer production. Proc Natl Acad Sci U S A 96, 1639-1644.

Swift, S., Winson, M. K., Chan, P. F. \& 11 other authors (1993). A novel strategy for the isolation of luxI homologues; evidence for the widespread distribution of a LuxR:LuxI superfamily in enteric bacteria. Mol Microbiol 10, 511-520.

Tokuda, M., Chen, W., Karunakaran, T. \& Kuramitsu, H. K. (1998). Regulation of protease expression in Porphyromonas gingivalis. Infect Immun 66, 5232-5237.

Williams, P., Camara, M., Hardman, A. \& 7 other authors (2000). Quorum sensing and the population dependent control of virulence. Philos Trans $R$ Soc Sect B 355, 667-680.

Winson, M. K., Swift, S., Fish, L., Throup, J. P., Jorgensen, F., Chhabra, S. R., Bycroft, B. W., Williams, P. \& Stewart, G. S. A. B. (1998). Construction and analysis of luxCDABE-based plasmid sensors for investigating $\mathrm{N}$-acylhomoserine lactone-mediated quorum sensing. FEMS Microbiol Lett 163, 185-192.

Winzer, K., Hardie, K. R., Burgess, N. \& 8 other authors (2002). LuxS: its role in central metabolism and the in vitro synthesis of 4-hydroxy-5-methyl-3(2H)-furanone. Microbiology 148, (in press).

Withers, H., Swift, S. \& Williams, P. (2001). Quorum sensing as an integral component of gene regulatory networks in Gram negative bacteria. Curr Opin Microbiol 4, 186-193.

Xie, H., Chung, W. O., Park, Y. \& Lamont, R. J. (2000). Regulation of Porphyromonas gingivalis fimA (fimbrillin) gene. Infect Immun 68, 6574-6579.

Yoneda, M. \& Kuramitsu, H. K. (1996). Genetic evidence for the relationship of Porphyromonas gingivalis cysteine protease and hemagglutinin activities. Oral Microbiol Immunol 11, 129-134.

Received 8 August 2001; revised 9 October 2001; accepted 18 October 2001. 